\title{
Research on Network Management of Mobile Communication Network based on Agent Technology
}

\author{
Yan-Fang Fu, Bing Cheng ${ }^{*}$, Peng-Hui Tian and Xiang Gao
}

Xi'an Technological University, School of Computer Science \& Engineering, xi'an710032, China

\begin{abstract}
This article is aimed at how to rapid configure communication equipment resource in the mobile communication network environment and puts forward a scheme including planning management, topology discovery, configuration management that is agent based network deployment management. Meanwhile it gives these modules' designs and implement methods. Experimental results show that we can improve the efficiency of the network deployment after using the mind of XML technology and intelligent agent, at the same time operating multiple network resources and multiple configuration management are also to be more intelligent in network configuration management.
\end{abstract}

Keywords: Agent technology, configuration management, XML technology.

\section{INTRODUCTION}

With the rapid development of mobile networks and network scale continually expanding, rapid configuration on network equipment and communication resources are receiving more and more attention. Configuration management was defined in [1] ISO/IEC7498-4: initializing and configuring the network, enabling it to provide network services. The aim is to achieve a particular functionality of network management and optimal network performance. Configuration management is responsible for monitoring and managing the entire network status and their connection relationship. The configuration information that it collects and monitors network devices is providing managers with querying the network running parameter and configuration status. Events generated by other function of network management or network commands of a manager automatically adjust the configuration of network devices to ensure the normal operation of the entire network.

The traditional mobile communication network configuration adopts full manual connection. To communicate with others units, users need to set the parameters by manually setting up each communication device. However especially for rapid deployment of the complex mobile network equipment, equipment for automatic management, real-time monitoring and other activities, this is a major defect.

This paper focuses on the design of agent technology whose application in network management are to be discussed. It will put forward a set of DHCP services technology and the application of agent technology.

\section{SYSTEM STRUCTURE OF THE MOBILE NET- WORK MANAGEMENT}

Mobile network communication deploy software is a software system that deploys and manages the communication network. According to the network of mobile communication network structure and the establishment of the force, deploy software adopts the layered and distributed management system structure with collaboration. According to the management functions, jurisdiction is divided into three layers : one-level network management center, two-level network management center and node network management (Fig. 1).

One-level network management center is deployed in the integrated communication vehicle. It is responsible for the entire network planning, network configuration and network topology. Meanwhile, it is also responsible for the

\section{SETUP DESIGN OF THE AGENT BASED MOBILE COMMUNICATION NETWORK}

\subsection{Network Planning Management}

Network planning process is a communication network design strategies, which is purposed to determine the key operation of mobile communication network design and technical issues, providing a perfect architecture framework. The planning consists of three phases: Firstly, Pre-planning stages. It is carried out before any planning implementation of a plan, which is used to determine useful resource types for applications. Secondly, Mission planning stage. The stage is completed on a task before the implementation of the plan. It needs to determine whether or not you can use the actual resources, as well as name an only ID in a database for each resource. Thirdly, Network operations planning stage. The stage is to be completed in the task execution. At this stage, you can see how the network expands in the task exe- 


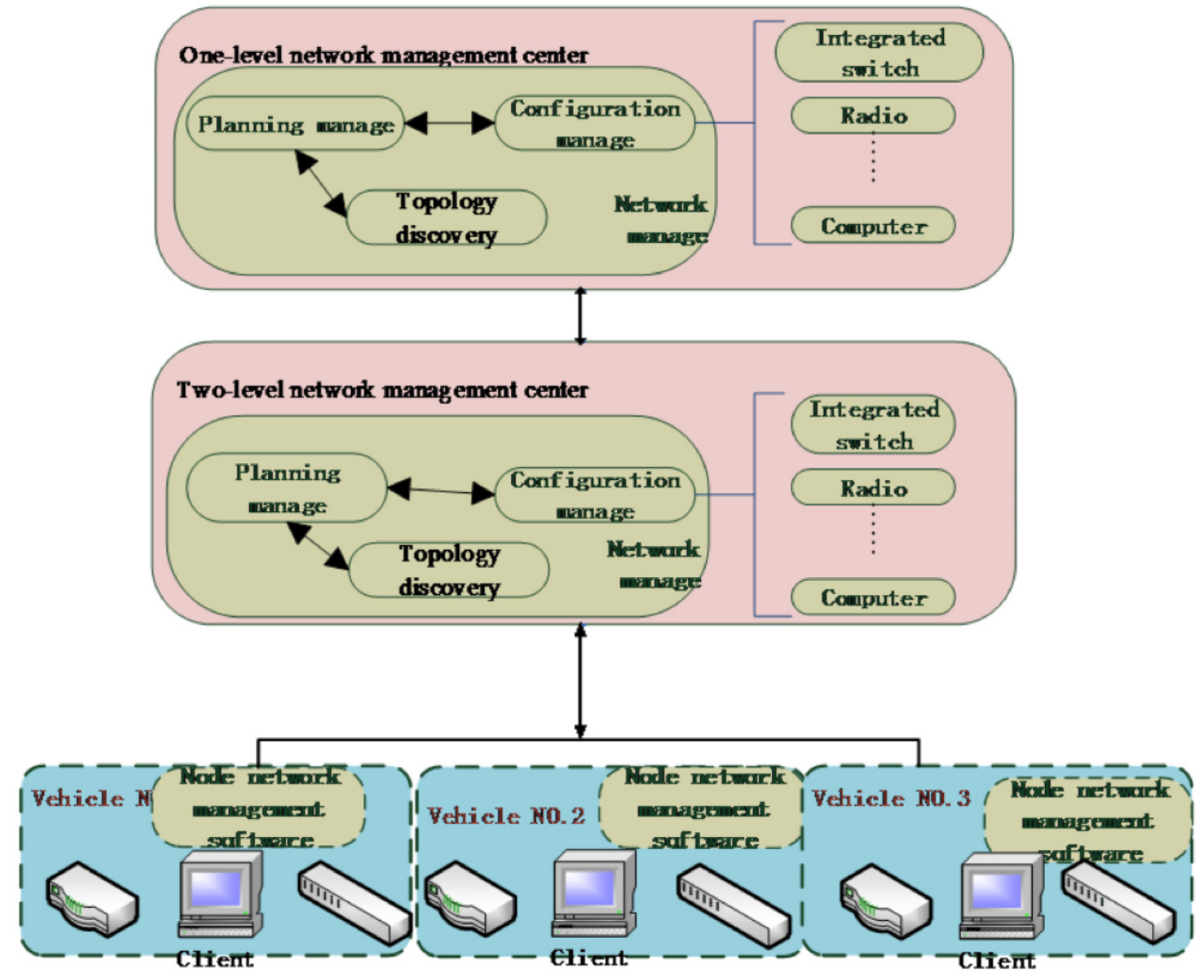

Fig. (1). System structure.

cution. Resource node can be based on the specific location of the deployment in the best possible service to users, and connection to the closest replay node in the communication network. In order to test node location and reliability of radio connections between nodes, you need to use a variety of analysis and validation tools [2]. Planning software receive planning documents through the system quickly and then set up communication equipment. According to the network architecture and communication needs, you should complete wireless subnet and link topology planning, including IP parameters, routing protocols, radio frequency, subnet, and working parameters [3]. Only through communication algorithm validate and the check of network planning in the plan for the integrity of your network topology, network parameters, correctness, consistency and effectiveness of the device parameters can you publish the configuration file, output network planning files and report files.

If the mobile network spread between moved network elements and moved the first node in common with same environment between fixed network elements and fixed the first node in the same, then in case of spreading the electromagnetic waves between the first node of the mobile network and moved network elements, the received signal value should be two fixed network along with the change of position between network elements in the first node and mobile reception the median level of the distribution of the sum. If in this propagation environment and two distributions with the same amplitude, the location of the standard deviation between moved network elements and moved the first node $\sigma_{L}$ is the square of two between fixed network elements and fixed the first node. If these calibration factor is shown below the Fig. (2). We can get a mobile network between moved network elements and moved the first node to the mathematical relationship between the probability of communication and system capacity in the condition of same propagation.

Then according to the basic idea of coverage forecast: based on propagation of radio wave propagation loss, bringing it into a wireless link in the maximum allowable path loss, coverage area, obtained subnet to the first node as the center of the circle formed by the established the circumference of the coverage area is how much power transmission, or reception. Considered from the angle of coverage is the key to select the radio wave propagation model, link budget model and transmission losses into the link budget equation.

So, based on battlefield covered by power control in a communication network planning process as shown in Fig. (3).

Here, we use the calculation method of coverage forecast to calculate transmitter power control problem in a combat scenario given the demand. Assume, two cars are apart from $4.8 \mathrm{~km}$, they are at the same speed relative to one another. 


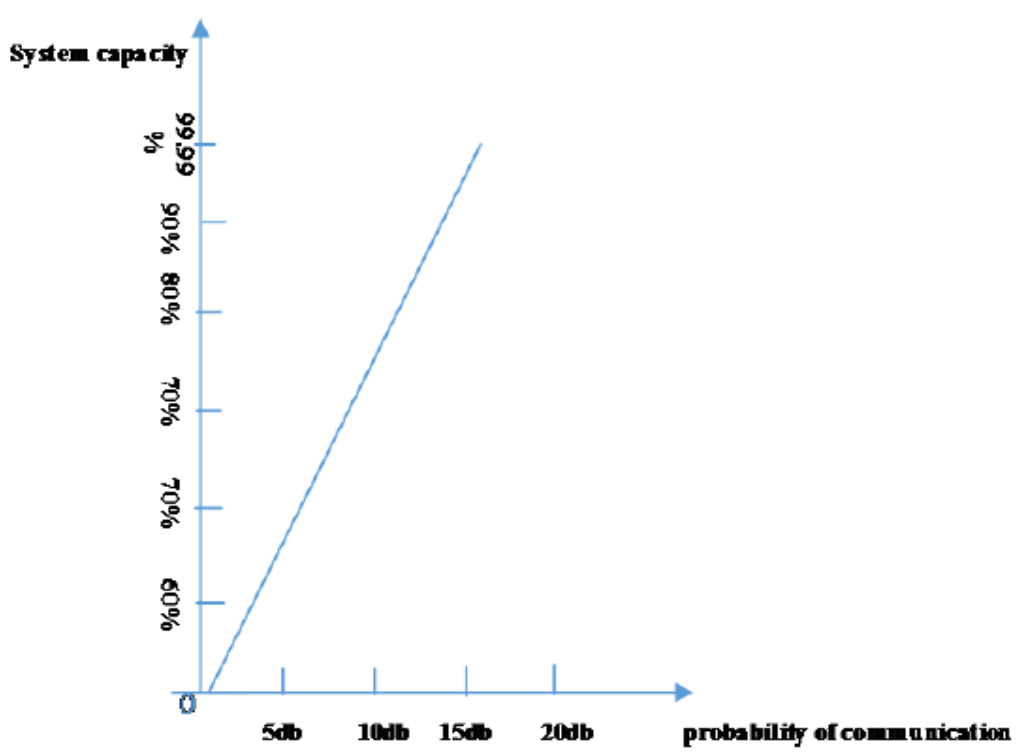

Fig. (2). In relationship of the probability of communication and system capacity.

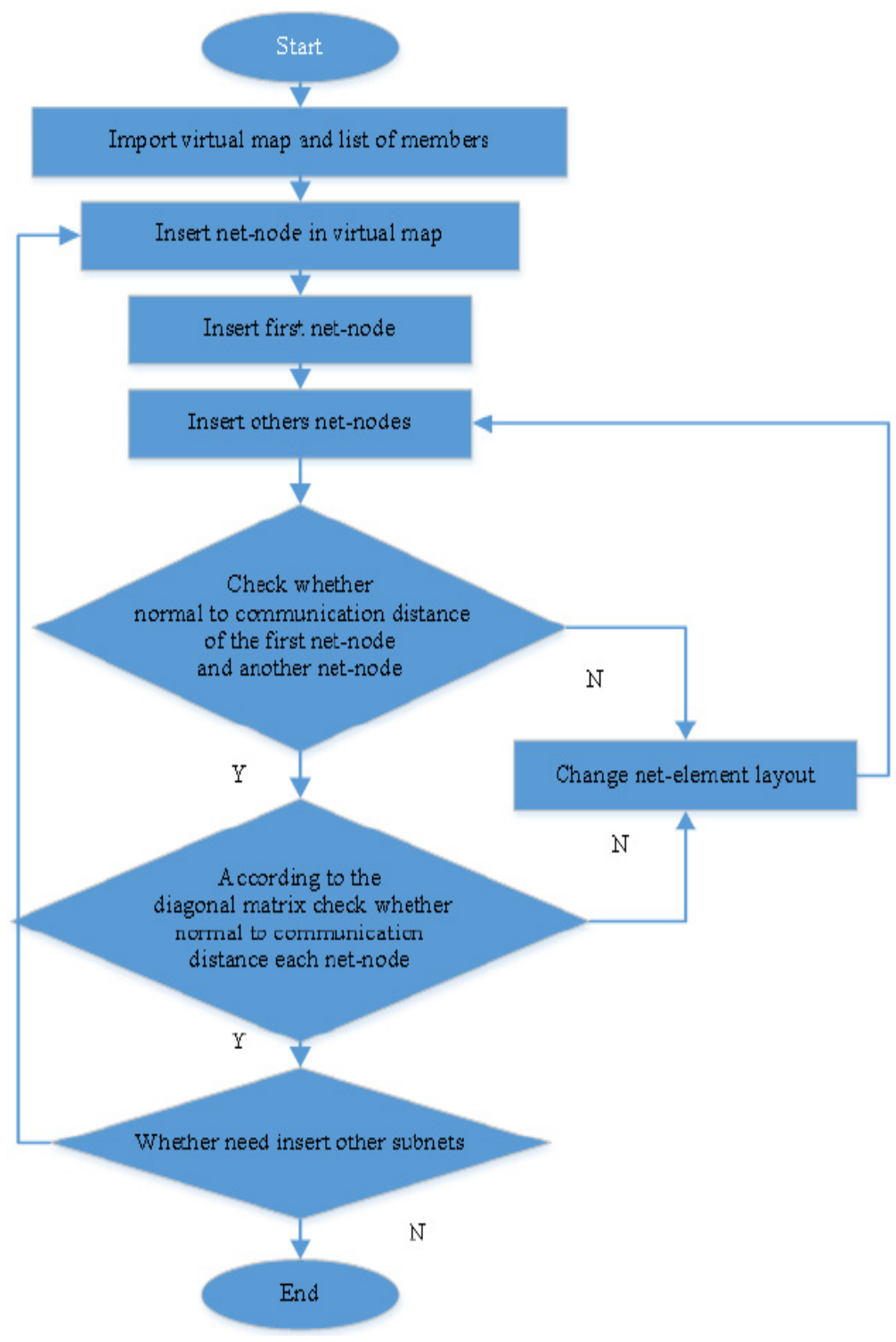

Fig. (3). Covering prediction process. 


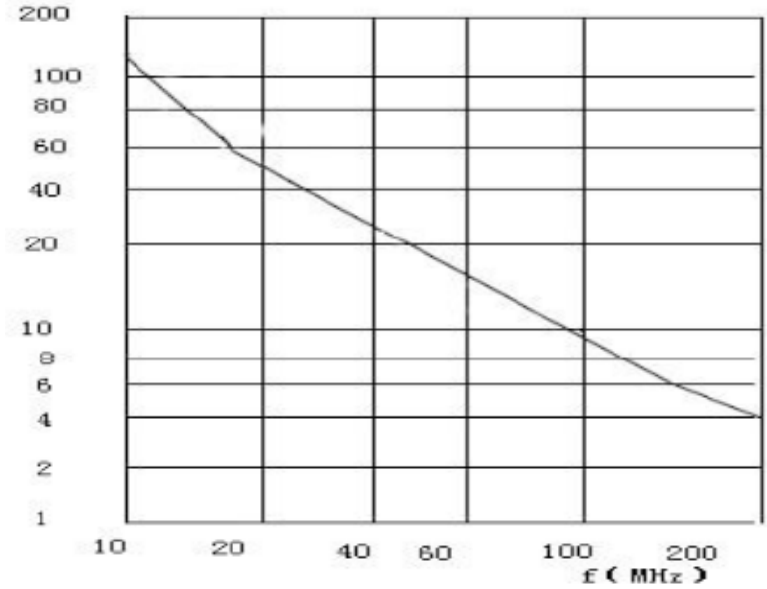

Fig. (4). The minimum effective antenna height in the great soil environment.

The communication mode is half-duplex, working in the $150 \mathrm{MHz}$ band. Ground wave height is $\Delta h=50 \mathrm{~m}$, the radio antenna height is $3 \mathrm{~m}$, feeder loss is $0.55 \mathrm{~dB}$, sensitivity of the receive device is $-104 \mathrm{~dB}$. The communication probability is $70 \%$, how much is needed for the transmitter power?

The calculation method is as follow: according to $f_{0}=150 \mathrm{MHz}, \Delta h=50 \mathrm{~m}$, standard deviation of the project tables to position is $\sigma_{L}=9 d B$. According to the standard deviation in a mobile case is the square of two as large as in a fixed case. $\sigma_{L}=12.7 \mathrm{~dB}, \mathrm{SM}$ (system margin) is $6 \mathrm{~dB}$ from Fig. (4). According to $f_{0}=150 \mathrm{MHz}$, we get the effective antenna height is $h_{0}=6 \mathrm{ft}$, but $h_{t}=9.8 \mathrm{ft}$. we calculate the median path loss $L_{M}=103 \mathrm{~dB}$.

According to $\Delta h=50 * 3.28=164 f t$, the terrain correction factor is $K_{h}=5 d B$ from Fig. (5).
dB

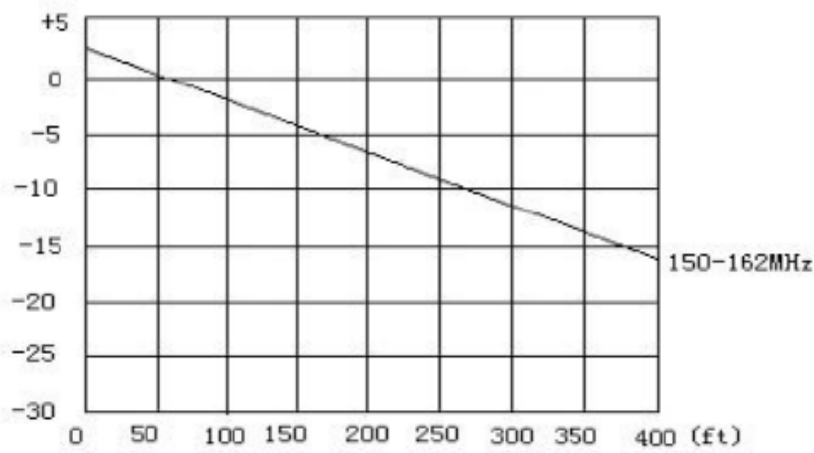

Fig. (5). The terrain correction factor.

According to the sending and receiving feeder loss of $0.5 \mathrm{~dB}$ and these calculation results, the link budget formula is as follow:

$P_{t}-L_{t}+G_{t}-S M-L_{M}+K_{h}+G_{r}-L_{r}=P_{r}$

So $P_{t}=P_{r}+L_{r}-G_{r}+S M+L_{M}-K_{h}-G_{t}+L_{t}$

$P_{t}=-104+0.5-2+103+5+6-2+0.5=7 d B \approx 5 W$

In these formulas, $P_{t}$ is the transmitter power; $P_{r}$ is receiver sensitivity; $L_{t} \backslash L_{r}$ is the transmitter $\backslash$ receiver feeder lin losses; $G_{t} \backslash G_{r}$ is the transmitter $\backslash$ receiver antenna gain.

According to this method, we can identify any two nodes within the subnet of any (including moved network elements and moved the first node) whether can be given in advance communication requirements to normal communication. so the above theory can be used to evaluate the backbone or any subnet planning covering the target at a lower level. For example Fig. (6) is a backbone of the command center levels of coverage predictions.

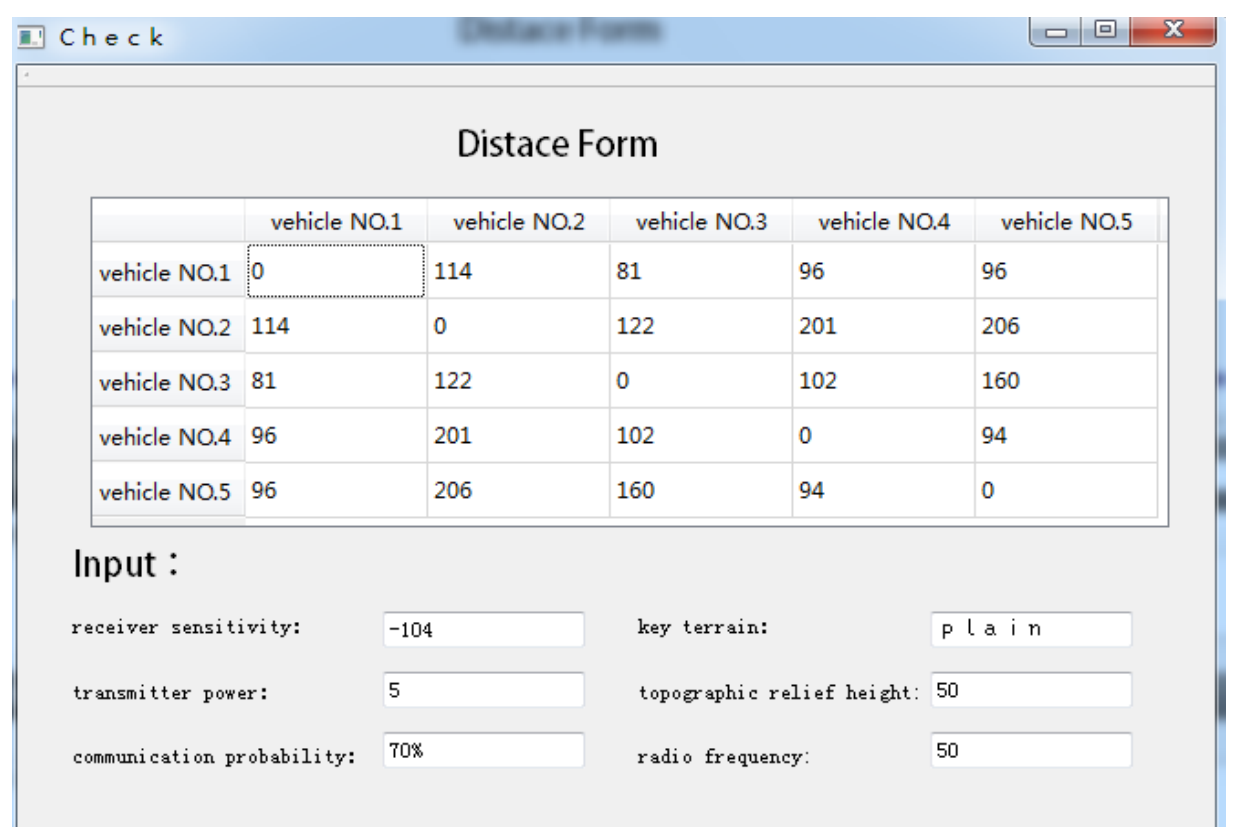

Fig. (6). Backbone of the command center levels of coverage predictions. 


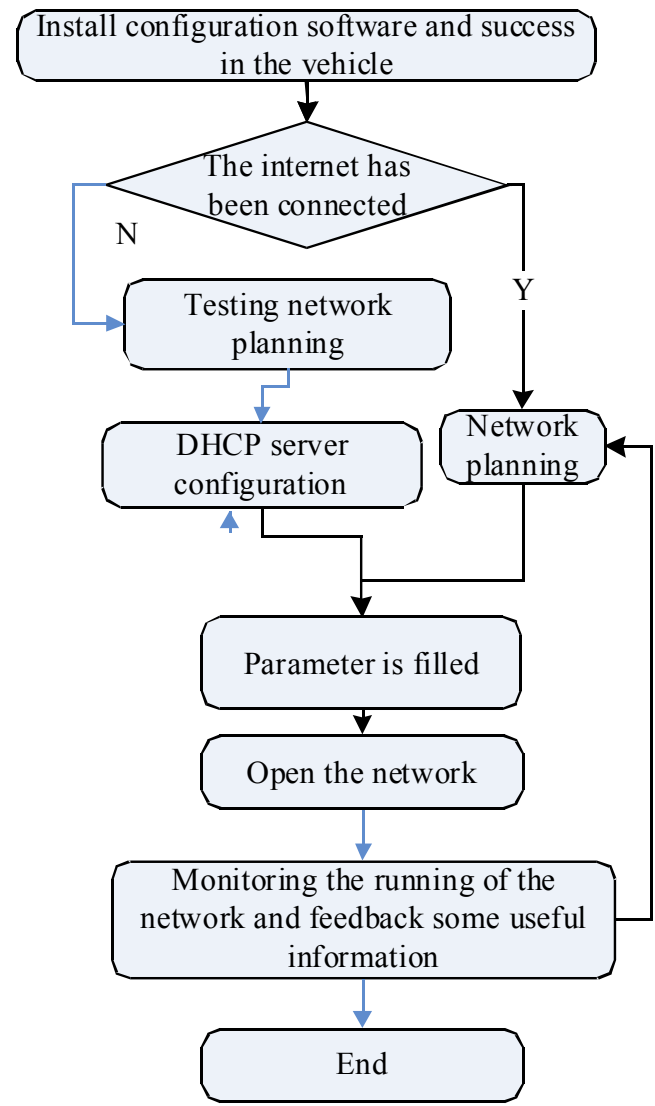

Fig. (7). Mobile network deployment of software typical process.

Here using diagonal matrix to represent the distance between each network, and according to the repository from the device and equipment parameters of the repository to read out the map, what is more, the operational requirement is communication of input, can predict whether standard for network coverage.

\subsection{Process of the Hardware in-Vehicle}

According to the typical scenario of communications network in-vehicle, mobile network deployment of software typical process is Fig. (7):

First, factory sets procedures to the vehicle, and installs the configuration software; second, it makes an ID for it; at last, it inputs the ID into the configuration software interface prompt box. If the network is not connected, it will start the server software with configuration software, the software starts the DHCP service to temporal IP in the particular network segment for entire in-vehicle host equipment. If the equipment has been connected, it will send the configuration files for each equipment, and then in-vehicle equipment will be connected each other again after receiving the file.

\subsection{Process of the International Mobile Network De- ployment}

In-vehicle communication network has been established, international mobile network deployment of software typical process is Fig. (8):

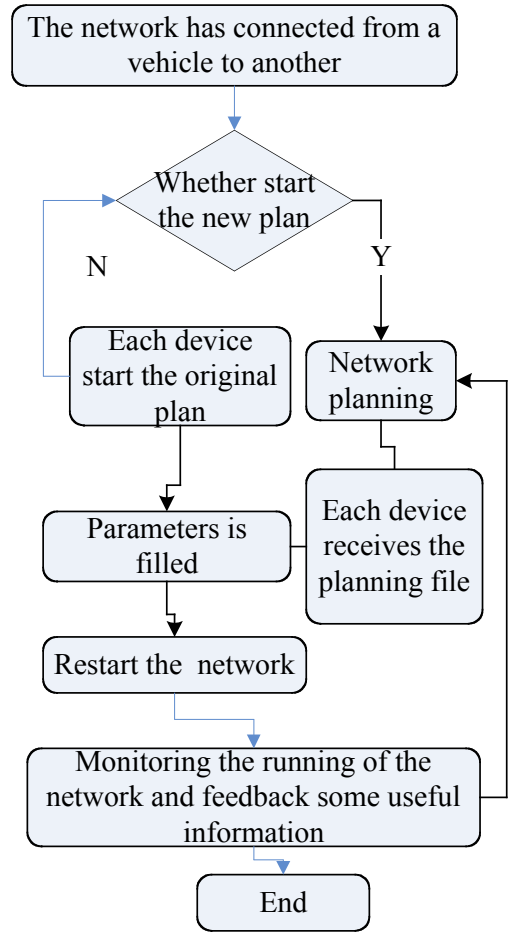

Fig. (8). International mobile network deployment of software typical process.

When we need to set up new IP for network equipment, first, we make a new configuration file by network planning software; second, broadcast the file for entire network equipment and each equipment will receive the file and find its own configuration file by ID; at last, restart the equipment.

\subsection{Process of the Network Monitor}

When entire equipment is connected with each other, start the network monitor software to detect network status. Then route the information acquired by SNMP protocol, complete the topology of the backbone, and for terminal device use the ICMP protocol monitor to check it is online or not.

In order to reduce the amount of network bandwidth, monitor software is used for hierarchical monitor. The monitor software with Two-level nodes only detect equipments and network status for this level and below level. Except for one-level node, topological relations among nodes will send to the superior in the form of files.

\section{THE MODULE DESIGN OF THE BASED CON- FIGURATION MANAGEMENT}

\subsection{Function Design}

Configuration management subsystem consists of the client and the server. Clients submit the configuration information to the server. The server boot remotely starts each agent to complete specific configuration based on planning information. Agent consists of three main modules: receiving module, sending module and configuring module. Receiving module is mainly responsible for receiving XML configuration information across the network, parsing XML files [4]. 


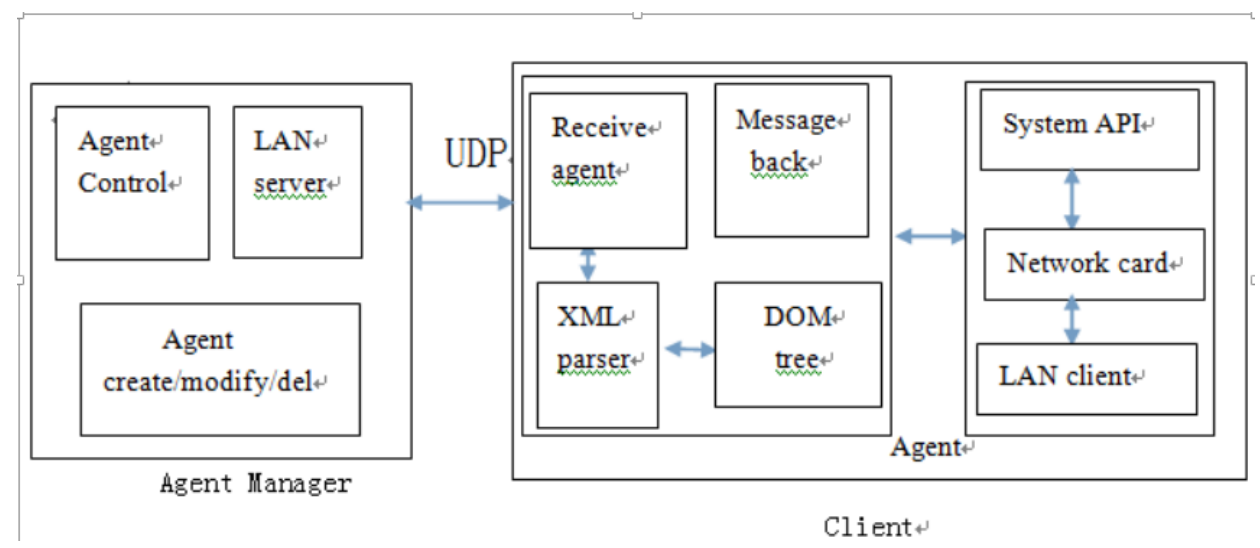

Fig. (9). Agent manager manages peer and subordinate agent.

Configuring module receives the parsed files to complete configuration for the equipment with ID. Sending module sends the current equipment status and configuration result of the operation to monitor subsystem in the form of the returning value. Different devices need have different information to be configured, based on the type of device configuration information and ID.

\subsection{Modules of Agent Network Configuration}

Management module is embedded in systems management server. As shown in Fig. (9), there are a number of modules. Agent manager manages peer and subordinate agent. Includes assigning tasks for agents, detect agents is functioning correctly. In order to detect managed device in time, agent control module receives data and information that a managed device is currently running from specific agents (message back). If users want to change current planning, agent managers will create a new agent for each device and delete the running agent, then insert the new agent for devices. Different devices are identified by ID numbers. At last the new agent sends information to agent control.

Planning information is stored in the form of XML files. Server broadcasts XML files in the network. Devices receive XML files by the receiving agent, then XML parser will deal with the file. Agent extracts its configuration information by the device ID. Next Change the device configuration information by system calls. At last, the message sends back the result of the alteration to servers.

The main tasks of the agent modules are to monitor and to configure the running on the managed network devices to ensure them to work correctly. Agent is send to the appropriate management of device in the form of programs to complete the configuration of network devices.

\section{REALIZATION OF THE SYSTEM}

\subsection{Creation of the XML File}

The XML is a Meta-Markup language. It can easily exchange the data between incompatible systems. Structure of the XML is a tree, from a root extending to its branches; its clear structure makes it easy to traverse management [5]. You can easily access to any element and element attributes. Using it you can store devices information and sent it to cor- responding devices, which provides a quick and accurate method.

Now I will describe our XML [6] structures and elements.

First of all, obtain a terminal physical address via a DHCP server and the device ID, then create the XML file. Key codes are as follows:

QDomDocument doc_confxml:

QDomElement root;

QDomText text_address;

QString strHeader("version=|"1.0।"encoding=|"UTF$8 \backslash " "$ );//XML standard file formats

doc_confxml.appendChild(doc_confxml.createProcessin gInstruction("xml", strHeader));

root=doc confxml.createElement("root");//create the root node

el_address=doc_confxml.createElement("mac");//create "MAC" element ].mac);

text_address $=$ doc_confxml.createTextNode(node_info $[\mathrm{i}$

stored a MAC of device into XML:

Part of the structure looks like this; where node00001 is the device ID.

$$
\begin{aligned}
& <\text { ?xml version="1.0" encoding="UTF-8"?> } \\
& <\text { root }> \\
& <\text { node } 0001> \\
& <\text { mac }>00: 50: 56: f 9: 63: 79</ \text { mac }> \\
& <\text { ip }>192.168 .205 .2</ \text { ip }> \\
& <\text { name }>\text { host_ } 1<\text { /name }> \\
& <\text { node } 0001> \\
& \quad \ldots \ldots \\
& </ \text { root }>
\end{aligned}
$$

Next network management unifies manually assign a unique IP for the device and keeps it in $<$ node00001 $>$ the element node. 
At last, DHCP server broadcasts the XML at the network and agent in the device processes the XML

\subsection{The Network Initialization of DHCP}

DHCP (Dynamic Host Configuration Protocol), adopts $\mathrm{C} / \mathrm{S}$ communication module. Clients put configurations to the server. Then servers return IP information and other configurations to clients. The study uses the address policy automatically assigned. It is great important to quickly build the connections between clients and servers [7]. DHCP server interface is shown in the following figure:

To start DHCP server, you can choose default IP or manual input IP for it. Chart data is some device information.

\subsection{Modifying the IP Address}

DHCP server sets up the connection for the first time between the network management centers and clients [8]. In order to achieve stability and real time communication between network management center and equipment, we need to change IP from dynamic assignment to static state. When the agent detects that the device is disconnected with the network management center, the agent will send messages to the network management center. The manager broadcasts XML files, mean while the agent accepts files and quickly resets the IP address of the device. This method reduces the time to reconnect and achieve stable communication in real time. Modifying the IP address is as follows:

1. First, DHCP server inputs a unique ID for the device. For example: node00001;

2. Manager broadcasts XML files, agent application reads the XML files and looks for node 00001 .

\subsection{Topology discovery}

At present, topology discovery bases on SNMP protocol as many network management product features. Such as HP Open view, IBM Tivoli and TRANSCEND 3COM. Other manufacturers also develop proprietary tools and protocols to discover the physical connection of the network..

To address the problem of large-scale network topology discovery, we use the SNMP protocol, read the standard SNMP MIB information, and solve the problem of multiple manufacturer devices. We analyze the existing network management software about the topology discovery and we set up the goal of the system topology discovery: (1) Fixing and Blocking: specify the beginning of topology discovery, and block the port of the specified IP address. (2) Parallel Speed-up: we use multiple threads in parallel to increase the speed of findings. (3) Conformance Checking: Compare with the result of a number of findings, and discover the network topology changes. Algorithms are as follows:

(1) Topology Discovery Algorithms: The algorithm receives topology discovery parameters, manages network devices in the discovery process, calls circularly router, switches devices and completes the specified depth of network topology discovery.

a. Check to reveal the depth and find the starting point;

b. Establish and discover databases; c. Discover starting point for device information and place it in the temporary router table;

d. Remove the temporary device in the routing table in order.

$\Theta$. If the type of device is a router, call the discover router to find out the router discovery method;

$\ominus$. If the temporary router discovery finishes it, but does not complete the intended depth, then go into the next cycle.

$\circledast$. If the type of device is a switch device, call it to find the switch discovery method;

(4). If the temporary switch is found, but doesn't complete the intended depth, then go into the next cycle.

(2) Router interface discovery algorithm: the met-hod is responsible for handling all routers to be found in a variety of information, and they are made to store in the appropriate database, and update the temporary router table; this algorithm use the first port as its unique identity. There is a range of valid IP address existing in the databases. The algorithm describes below:

a. Get the first port on the router's physical address;

b. If the router is already in the database, it will be removed the temporary router table;

c. Get all kinds of information about ports;

d. If the IP address of the port with this router has more than $50 \%$ in common with IP address of port with other routers, go to the "b"step.

e. Obtain all kinds of information for router, and plug it into the router table.

f. Delete the router from the temporary router table, and then insert the port into the interface table, and update the temp-orary databases.

\section{CONCLUSION}

Design and implementation of this article are based on mobile agent technology about the mobile network management solution. According to the network architecture, communication needs, your wireless network should link topology planning,; through correspondence algorithm for testing the integrity of the topology, then finally release the configuration file, output report file and network planning documents. When a client needs to communicate with the server, the agent application is activated and receives XML files from the reserve, then parses the file and changes synchronously the configuration of the device in real-time. When the agent application is performing operations, and the client is not interrupted, thus the client can modify the configuration files and reduce the burden on the client, improving the ability of the client's parallel, and the design of mobile communication device on the network can do multiple management operations to make network management more intelligent.

\section{CONFLICT OF INTEREST}

The authors confirm that this article content has no conflict of interest. 


\section{ACKNOWLEDGEMENTS}

Declared none.

\section{REFERENCES}

[1] G. Chen, Q. Kong, and J. Etheridge, "Integrated TMN service management", Journal of Network and Systems Management, vol. 7, no. 4, pp. 469-493, 1999.

[2] G. Schürmann, "The evolution from open systems interconnection (OSI) to open distributed processing (ODP)", Computer Standards \& Interfaces, vol. 17, no. 1, pp. 107-113, 1995.

[3] S. Murthy, and A. Vernekar, "TMN-Based interoperability network administration interface", Journal of Network and Systems Management, vol. 3, no. 2, pp. 217-227, 1995.
[4] S. Ahn, and J. C. Wook, "A study upon SNMP MIB II algorithm: for the calculation of the line utilization", The Journal of Supercomputing, vol. 33, no. 1, pp. 79-91, 2005.

[5] M. Daniele, and B. Wijnen, Agent Extensibility(AgentX)Protocol Version 1, 2000.

[6] D. Thomas, "Algorithms and architectures for dataprivacy", $\mathrm{PhD}$ thesis, Stanford University, 2007.

[7] B. V. S. N. Sushma, "Multi-level data privacy for mobile databases using multi-views", $\mathrm{PhD}$ thesis, Texas A\&M University Kingsville, 2010

[8] J. Colmenero, A. Arbe, and F. Alvarez, "Hydrogen motions and the $\alpha$-relaxation in glass-forming polymers: Molecular dynamics simulation and quasi-elastic neutron scattering results" Pramana, vol. 63, no. 1, pp. 25-32, 2004.

(C) Yan-Fang et al.; Licensee Bentham Open.

This is an open access article licensed under the terms of the (https://creativecommons.org/licenses/by/4.0/legalcode), which permits unrestricted, noncommercial use, distribution and reproduction in any medium, provided the work is properly cited. 